\title{
Importância da neuroestimulação medular no tratamento da síndrome do insucesso da cirurgia espinal
}

\author{
Isabela Palmeira Gomes ${ }^{1}$, Otaviano Ottoni da Silva Netto², \\ Rodrigo Almeida Matos ${ }^{2}$, Ledismar José da Silva ${ }^{3}$ \\ Pontifícia Universidade Católica de Goiás (PUC-Goiás), Goiânia, GO, Brasil.
}

\section{RESUMO}

Eletrodos vêm sendo utilizados desde 1967 para estimulação da coluna espinal (em inglês, spinal cord stimulation - SCS) no tratamento de dor crônica refratária em uma série de distúrbios dolorosos, entre eles a síndrome do insucesso da cirurgia espinal (em inglês, failed-back surgery syndrome - FBSS), caracterizada por dor persistente após cirurgias de coluna, principalmente laminectomia. Neste artigo, apresenta-se uma revisão sistemática da literatura sobre o estudo da neuromodulação no tratamento da FBSS, utilizando-se as plataformas dos portais virtuais PubMed e MedLine, com o objetivo de levantar evidências que corroborem a eficácia e a segurança desse procedimento para dor crônica lombar decorrente de FBSS. A seleção dos estudos, publicados no período entre janeiro de 2003 e janeiro de 2013, envolveu critérios de análise de eficácia (melhora da dor, com redução de $50 \%$ ou mais, utilizando a Escala Visual Analógica - VAS - ou outras similares) e de segurança (quando complicações foram citadas). Foram inicialmente identificados 186 artigos, entre os quais nove foram selecionados por preencherem os critérios de inclusão/exclusão, totalizando 313 pacientes. Em todos os trabalhos selecionados, observou-se melhora considerável da dor após os procedimentos neuromodulatórios. Conclui-se que, quando bem indicada, a SCS é mais eficaz do que a reoperação ou qualquer outro tipo de terapia conservadora no tratamento de dor crônica lombossacral.

\section{PALAVRAS-CHAVE}

Neurocirurgia, medula espinal, dor crônica, dor neuropática.

\section{ABSTRACT}

Importance of spinal cord stimulation for the treatment of failed back surgery syndrome Electrodes have been used since 1967 for spinal cord stimulation (SCS) for the treatment of chronic pain in a series of painful conditions, such as failed-back surgery syndrome (FBSS), characterized by persistent pain after surgical procedures in the spinal column, mainly laminectomy. In this article, a systematic review of the literature is presented about the study of neuromodulation for the treatment of FBSS using data available at PubMed and MedLine, aiming to assess evidences that corroborate the efficacy and safety of this procedure for the treatment of low back chronic pain due to FBSS. The selection of the articles, published from January 2003 to January 2013, involved criteria of efficacy analysis (pain relief, with reduction by $50 \%$ or more, using the visual analogue scale - VAS or other similar ones) and safety (when complications were mentioned). Initially, 186 articles were identified, among which, nine were selected because they fulfilled the inclusion/exclusion criteria, in a total of 313 patients. In all the articles selected, considerable pain relief was observed after neuromodulation procedures. We conclude that, when correctly indicated, SCS is more efficient than a new surgery or any other type of conservative therapy for the treatment of low back chronic pain.

\section{KEYWORDS}

Neurosurgery, spinal cord, chronic pain, neuropatic pain.

1 Graduando em Medicina pela Pontifícia Universidade Católica de Goiás (PUC-Goiás), Goiânia, GO, Brasil.

2 Médico, clínico geral, pela PUC-Goiás, Goiânia, GO, Brasil.

3 Neurocirurgião, professor da PUC-Goiás, Goiânia, GO, Brasil. 


\section{Introdução}

O uso de estimulação elétrica por meio de eletrodos foi introduzido em 1967, por Shealy et al., ${ }^{1}$ os quais utilizaram a neuroestimulação espinal dorsal para tratamento de dor crônica refratária. A partir de então, a técnica vem sendo utilizada no tratamento de diversos distúrbios dolorosos, como aracnoidite, ${ }^{2}$ dor de membro fantasma, ${ }^{3}$ esclerose múltipla, ${ }^{3,4}$ doença vascular periférica, ${ }^{5}$ tumores, ${ }^{3,6}$ lesões de plexo braquial, ${ }^{3,7}$ neuralgia pós-herpética, ${ }^{5,8}$ dor por isquemia periférica, ${ }^{9,10}$ síndrome da dor complexa regional (em inglês, complex regional pain syndrome - CRPS), ${ }_{11}^{11}$ neuropatia diabética ${ }^{12}$ e síndrome do insucesso da cirurgia espinal. ${ }^{13}$

De acordo com a International Association for the Study of Pain (IASP, Associação Internacional de Estudo da Dor), a síndrome do insucesso da cirurgia espinal (em inglês, failed-back surgery syndrome - FBSS), também chamada por alguns autores de síndrome pós-laminectomia (SPL), é definida como dor lombar espinal de origem desconhecida que persiste na mesma localização da dor original, apesar das intervenções cirúrgicas, ou que se instala após as cirurgias. ${ }^{14} \mathrm{~A}$ lombalgia pode ou não se associar à dor referida ou irradiada.

Nesses casos, o alívio da dor pode ser propiciado por meio de impulsos elétricos em fibras que podem inibir a condução de sinais dolorosos para o cérebro, de acordo com a Teoria do Portão proposta por Melzack e Wall ${ }^{9}$. O efeito simpaticolítico da estimulação da coluna espinal (em inglês, spinal cord stimulation - SCS) é considerado responsável pela eficácia do tratamento modulatório, geralmente por não haver eliminação da dor, mas modificação de sua percepção. ${ }^{13}$

Estima-se que entre 60\% e 85\% das pessoas em geral experimentarão dor lombossacral pelo menos uma vez na vida, e em $10 \%$ a $20 \%$ desses casos a dor lombar se tornará crônica, necessitando de algum tipo de intervenção prolongada. ${ }^{15,16}$ Avalia-se que haja a realização de mais de 300 mil laminectomias por ano nos Estados Unidos, com taxa de insucesso de $20 \%$ a $40 \% .^{17}$

Considerando o número expressivo de pacientes que desenvolvem a FBSS, os crescentes gastos públicos e privados com tratamentos decorrentes disso e a repercussão negativa na qualidade de vida desses indivíduos, entendeu-se ser necessária uma revisão da literatura com o intuito de avaliar a segurança e a eficácia da neuroestimulação medular no tratamento da FBSS.

\section{Materiais e métodos}

Para a consecução deste trabalho, foi realizada revisão sistemática da literatura internacional, abarcando publicações pertinentes sobre a temática pesquisada, por intermédio da plataforma dos portais virtuais PubMed e MedLine, no período de janeiro de 2003 a janeiro de 2013. Foram considerados como sujeitos desta pesquisa pacientes submetidos à técnica de estimulação da coluna espinal (em inglês, spinal cord stimulation - SCS) para tratamento de dor crônica lombar decorrente de FBSS.

A seleção dos artigos pertinentes iniciou-se com a busca nas referidas plataformas utilizando os seguintes descritores em português, bem como suas combinações e os respectivos termos em inglês: "dor crônica"; "dor crônica refratária"; "terapia de estimulação elétrica"; "estimulação elétrica da coluna espinal"; "implante de gerador de pulso/estimuladores"; "síndrome pós-laminectomia (SPL)"; "laminectomia"; "síndrome do insucesso da cirurgia espinal (FBSS)"; "tratamento de dor crônica".

Para a utilização dos dados obtidos, as publicações foram submetidas a filtros de inclusão e exclusão, atendendo a critérios preestabelecidos de segurança $\mathrm{e}$ eficácia. Não foram analisadas neste estudo técnicas, posicionamento, localização do implante de eletrodos ou modelos específicos de neuroestimuladores.

Para serem incluídos, os artigos deveriam preencher os seguintes critérios: ser publicados em inglês ou português; abordar casos de pacientes com dor em coluna lombar após intervenção cirúrgica; apresentar porcentagens e/ou estatísticas relatadas pelos autores e disponíveis; apresentar avaliação da eficácia e/ou segurança do SCS; disponibilizar avaliação da dor realizada por meio de escala visual analógica (VAS) ou outras escalas símiles, apresentando $50 \%$ ou mais de redução da dor em uma escala de pontos; declarar o número de pacientes estudados; executar follow-up após SCS de pelo menos seis meses.

Foram excluídos da avaliação artigos: sobre revisão sistemática de literatura; que não especificassem número de pacientes ou resultados claros e específicos da FBSS ou SPL; que incluíssem outras espécies que não a humana; que incluíssem pacientes que receberam implantes no período entre 2003 e 2013.

Empregando a sequência descrita, inicialmente foram identificados 186 artigos, dos quais apenas nove preencheram todos os critérios de inclusão e exclusão. Dessa maneira, foi incluído neste estudo um total de 313 pacientes submetidos à SCS para tratamento da FBSS (Tabela 1). 


\begin{tabular}{|c|c|c|c|c|c|c|c|c|}
\hline Estudo & $\begin{array}{c}\text { Ano de } \\
\text { publicação }\end{array}$ & Local & $\begin{array}{c}\text { Participantes } \\
\left(\mathbf{n}^{\circ}\right)\end{array}$ & $\begin{array}{l}\text { Design do } \\
\text { estudo }\end{array}$ & Idade (anos) & $\begin{array}{c}\text { Tempo de } \\
\text { follow-up } \\
\text { (meses) }\end{array}$ & $\begin{array}{c}\text { Melhoria da } \\
\text { intensidade } \\
\text { da dor - VAS }{ }^{1} \\
\text { (mais de } 50 \% \text { ) }\end{array}$ & $\begin{array}{c}\text { Satisfação do } \\
\text { paciente - QOL } \\
(6 \text { meses })\end{array}$ \\
\hline Blond et al. ${ }^{19}$ & 2004 & $\begin{array}{c}\text { França } \\
\text { (multicêntrico) }\end{array}$ & 53 pacientes & $\mathrm{RCT}^{3}$ & $50,7(32-78)$ & 6 a 24 & $\begin{array}{c}6 \text { meses: redução } \\
60,9 \% ; 24 \text { meses: } \\
60 \% \text {; VAS } \\
\text { melhora de } 70 \% \\
\text { (inicial } 7,99 / 10 \text {, } \\
\text { final } 3,12 / 10 \text { ) }\end{array}$ & Geral $40 \%$ \\
\hline North et al..$^{18}$ & 2005 & Estados Unidos & $\begin{array}{c}\mathrm{SCS}^{4}-19 \\
\text { pacientes } \\
\text { Controle }-26\end{array}$ & RCT & $50,2(36-65)$ & 6 a 36 & $\begin{array}{l}\text { SCS 9/19 }(48 \%) ; \\
\text { Controle } 3 / 26 \\
\quad(11,5 \%)\end{array}$ & $\begin{array}{c}\text { SCS } 5 / 24 \\
(21 \%) ; \text { controle } \\
14 / 26(54 \%)\end{array}$ \\
\hline Kumar et al. ${ }^{20}$ & 2007 & $\begin{array}{c}\text { Austrália } \\
\text { e Europa } \\
\text { (multicêntrico) }\end{array}$ & $\begin{array}{c}\text { SCS }-50 \\
\text { pacientes } \\
\text { Controle }-44\end{array}$ & RCT & $52(42-63)$ & 6 a 12 & $\begin{array}{c}\text { SCS 24/50 (48\%); } \\
\text { controle 4/44 (9\%) }\end{array}$ & $\begin{array}{c}\text { SCS } 33 / 50 \\
(66 \%) ; \text { controle } \\
8 / 44(18 \%)\end{array}$ \\
\hline $\begin{array}{l}\text { Coleman e } \\
\text { Mackey }^{26}\end{array}$ & 2009 & Estados Unidos & $\begin{array}{c}\text { SCS }-48 \\
\text { pacientes } \\
\mathrm{CMM}^{5}-48 \\
\text { pacientes }\end{array}$ & RCT & $\begin{array}{c}\text { Não } \\
\text { declarado }\end{array}$ & 6 & $\begin{array}{c}\text { SCS }(48 \%) ; \\
\text { controle CMM } \\
(9 \%)(p<0,001)\end{array}$ & SF-36 - 7 de $8^{* *}$ \\
\hline $\begin{array}{l}\text { Vonhögen } \\
\text { et al. } .^{27}\end{array}$ & 2011 & Holanda & 20 pacientes & Retrospectivo & $52,15(33-81)$ & 6 a 12 & $\begin{array}{c}\text { SCS }-45,7 \% \\
\text { (dor lombar); } \\
55 \%(\mathrm{MMII}){ }^{6} \\
\text { clinicamente }-85 \%\end{array}$ & $\begin{array}{l}66 \% \text { satisfação } \\
\text { geral; } 70 \% \\
\text { melhora clínica }\end{array}$ \\
\hline Kinfe et al..$^{21}$ & 2012 & Alemanha & 81 pacientes & Prospectivo & $57(27-82)$ & 6 a 12 & $\begin{array}{c}73 \% \text { VAS } 2.3 \\
\text { (preop 8.4) }\end{array}$ & Não declarado \\
\hline $\begin{array}{l}\text { Rigoard } \\
\text { et al. }\end{array}$ & 2012 & França & 11 pacientes & Observacional & $49,9(31-72)$ & 6 & $\begin{array}{c}\text { SCS 9/11 (81,8\%). } \\
\text { VAS global: } 2,22 \\
\text { (8,2 pre-op); } \\
\text { VAS lombar: } \\
\text { 1,5 (7,8 pre-op); } \\
\text { VAS MMII: } 0,5 \\
\text { (7,6 pre-op) }\end{array}$ & $\begin{array}{l}80 \% \text { satisfação } \\
\text { geral }\end{array}$ \\
\hline $\begin{array}{l}\text { De Andres } \\
\text { et al. }{ }^{17}\end{array}$ & 2013 & Espanha & 10 pacientes & Relato de caso & $53,6(28-78)$ & 12 & $71,40 \%$ & Não declarado \\
\hline Logé et al. ${ }^{29}$ & 2013 & Bélgica & 21 pacientes & Prospectivo & $57(33-76)$ & 6 a 40 & $\begin{array}{c}\text { FU- } 1^{7} \text { e FU-2 - } \\
62,52 \% \\
\text { VAS - } 75,79 \%\end{array}$ & $\begin{array}{c}85,3 \% \text { satisfação } \\
\text { geral }\end{array}$ \\
\hline
\end{tabular}

VAS: Escala Visual Analógica; QOL: qualidade de vida; RCT: ensaio randomizado controlado; SCS: estimulação da coluna espinal; CMM: tratamento médico convencional; MMII: membros inferiores; FU: follow-up.

selecionados para o presente estudo nove artigos, os quais incluíram 313 resultados de pacientes com dor crônica por FBSS. Foram observados resultados em seis meses de follow-up e, quando possível, em períodos posteriores.

As características de todos os estudos incluídos estão descritas na tabela 1. Entre os nove artigos selecionados, quatro são ensaios randomizados controlados, entre os quais dois são estudos multicêntricos, um é estudo observacional, um é estudo retrospectivo, dois são estudos prospectivos e um é relato de caso.

Observou-se predomínio do sexo feminino nos artigos avaliados, alcançando um total de $53 \%$ dos pacientes incluídos nos estudos em que o sexo foi declarado. A média de idade foi de 52,46 anos, havendo pacientes entre 19 e 83 anos de idade.

$\mathrm{Na}$ análise de intensidade da dor, utilizando-se a VAS comparativa entre o momento de definição e o rastreamento para a intervenção e dos primeiros seis meses após o implante, todos os estudos evidenciaram melhora significativa do padrão da dor previamente apresentada. No estudo espanhol de De Andres et al. ${ }^{17}$, com 10 pacientes, entre os quais sete apresentaram um percentual de $60 \%$ de satisfação e melhoria da dor superior a $50 \%$ no VAS, apenas três pacientes não apresentaram melhora significativa e foram exatamente os que apresentavam dor irradiada em região perineal, confirmando dados da literatura levantada - Hunter et $a l .{ }^{16}$ citaram que não há melhora significativa da dor crônica em região pélvica.

No estudo de North et al.$^{18}$, os pacientes submetidos à SCS foram significativamente menos propensos à reintervenção cirúrgica do que o grupo controle ( 5 dos 24 pacientes versus 14 dos 26 controles, $p>0,02$ ). Ainda no mesmo estudo, foram avaliadas as descrições sobre satisfação dos pacientes, em que os padrões apresenta- 
dos foram superiores a $21 \%$, sendo o único dado abaixo de $40 \%$ entre todos os estudos avaliados.

Nos casos de estudo controle, quando comparados os grupos de intervenção modulatória, a taxa média de satisfação ultrapassou a do controle, sendo significativamente maior ( $p>0,001)$ e apontando uma percepção com resposta significativa na qualidade de vida por parte dos pacientes, bem como casos de retorno a atividades laborais.

Embora não tenha sido parte dos objetivos deste trabalho e de a maior parte dos estudos avaliados não ter relatado complicações, por não fazer parte do escopo do estudo ou por não ter ocorrido, mas apenas como adendo, as complicações relatadas encontradas no pós-operatório foram: migração do eletrodo, infecção, hemorragia peridural, seroma, hematoma, perda de liquor, paralisias e parestesias, dor no local do implante, falha da estimulação, reações alérgicas, erosão da pele, mau funcionamento do estimulador (falha de software, hardware ou bateria) e fios/cabos soltos ou deslocados.

O estudo de Blond et al. ${ }^{19}$ mostrou que a intensidade geral da dor em VAS diminuiu. Em termos de intensidade da dor neuropática (unilateral ou bilateral ciática), os níveis de alívio obtidos foram bons (em média, de $64,8 \%$ em seis meses, $55,6 \%$ em 12 meses e $60 \%$ em 24 meses). A intensidade da dor foi reduzida de forma muito significativa $(\mathrm{p}<0,01)$, apresentando taxa de sucesso, em percepção dos pacientes, de 70\% em seis meses e de $50 \%$ em 24 meses.

No estudo de Kumar et al. ${ }^{20}$, comparando a SCS e o tratamento por meio de medicamentos, 24 pacientes do grupo de SCS (48\%) e quatro do grupo de tratamento medicamentoso exclusivo (9\%) obtiveram resultados primários de $50 \%$ de alívio da dor $(\mathrm{p}<0,001)$, evidenciando que a SCS é mais eficaz. Na análise após 12 meses, houve resposta positiva de $34 \%$ dos pacientes submetidos a SCS em comparação com $7 \%$ do grupo de tratamento medicamentoso exclusivo $(\mathrm{p}=0,005)$, mantendo o padrão de maior eficácia da SCS.

Os dados mais positivos mostraram resultados clínicos para melhor cobertura da parestesia em 87\% entre 81 pacientes e uma média considerável de redução da dor de 73\% (pré-operatório VAS 8,4 e VAS pós-operatório 2,3 nos 12 meses de follow-up), no trabalho de Kinfe et al. ${ }^{21}$.

\section{Discussão}

Vários estudos concluíram que existem algumas evidências de efeitos positivos do tratamento por meio da SCS em alguns diagnósticos de dor crônica, embora ainda exista necessidade de conduzir mais estudos randomizados..$^{22-24} \mathrm{Em}$ todos os estudos incluídos no presente trabalho, os autores relataram melhora na dor. ${ }^{17,19-21,25-29}$ Os resultados mostrados pela série de casos, contudo, são mais positivos do que os resultados de ensaios clínicos randomizados.

$\mathrm{O}$ estudo de $\mathrm{Kumar}^{30}$ forneceu evidências sobre os efeitos da SCS em melhoria da qualidade de vida e estado funcional dos pacientes. A SCS parece oferecer mais perspectiva de retorno às atividades laborais (15\% para SCS e $0 \%$ para o grupo controle), o que também afeta a relação custo-benefício dos resultados. ${ }^{24,31} \mathrm{O}$ impacto da SCS sobre a depressão foi relatado em apenas uma série de casos e mostrou melhorias significativas entre antes e após o procedimento. ${ }^{20}$

Os ensaios clínicos randomizados de Turner et al. ${ }^{24}$ e Kumar et al. ${ }^{31}$ mostraram maior satisfação do paciente com a SCS do que com o tratamento controle, seja em termos de satisfação com o alívio da dor, seja pela avaliação por meio da escala de qualidade de vida (QOL) realizada pelos pacientes.

Alguns estudos relataram certas complicações, em sua maioria, problemas técnicos associados com o mau funcionamento dos eletrodos, deslocamento ou outra falha de hardware e infecções. ${ }^{23,24,32}$

O relatório da Health Technology Assessment (HTA - Canadá) resume os resultados de diversos estudos: problemas com o eletrodo foram relatados a partir de $4 \%$ a $42,8 \%$; problemas relacionados com o gerador de pulso, de 1,6\% a 6\%; problemas de extensão do cabo, de $10 \%$; reoperação, de $11 \%$ a $50 \%$; dissecção subcutânea de bolso para o gerador, de $8,3 \%$; infecçã,o de $1,4 \%$ a $11,7 \%$; e fístula liquórica, de $0,3 \%$ a $7 \% .^{25}$

De acordo com a literatura, complicações biológicas (principalmente infecções) são mais comuns nos primeiros três meses após o implante de eletrodos medulares, embora geralmente pequenas e reversíveis. ${ }^{33,34}$

Embora o número de estudos controlados como a temática específica seja restrito, bem como uma compreensão do mecanismo exato pelo qual a SCS produz o seu efeito, esta se tornou uma terapêutica indispensável. Constitui ferramenta promissora para o tratamento de muitas condições de dor crônica e tem muitas vantagens sobre outras terapias propostas para o tratamento de FBSS.

Os eletrodos da SCS podem ser removidos a qualquer momento, se necessário, causando pouco desconforto para o paciente, diferente das técnicas ablativas. Em longo prazo, em contraste com os inúmeros efeitos colaterais sistêmicos de medicamentos orais, não existem efeitos secundários em decorrência da utilização da SCS. $23,25,35$

A má indicação de intervenção espinal é a maior responsável pela FBSS, podendo ter até $30 \%$ de falha. ${ }^{23}$ Deve-se lembrar que, apesar do sucesso evidenciado 
em todos os estudos analisados quanto ao tratamento da FBSS com o uso da SCS, os pacientes geralmente não deixam de utilizar medicamentos para alívio da dor, embora haja diminuição significativa tanto das doses quanto das quantidades de drogas empregadas nesse controle..$^{23,34,36}$

\section{Considerações finais}

Como evidenciado nos estudos levantados, a SCS é mais eficaz do que a reoperação como tratamento para a dor crônica lombossacral ou do que qualquer outro tratamento conservador, quando bem indicada. Observou-se que $61,4 \%$ dos pacientes submetidos à SCS apresentaram resultados considerados positivos, com melhora superior a 50\% na avaliação da VAS após intervenção modulatória nos primeiros seis meses.

Houve evidência de melhora significativa, de acordo com a satisfação dos pacientes submetidos à SCS, bem como na qualidade de vida analisada, algumas vezes recuperando o potencial laborativo dos indivíduos. Por intermédio do QOL, 63,61\% dos pacientes submetidos à SCS consideraram que houve melhoria da sua qualidade de vida após a intervenção.

A neuromodulação mostrou-se eficaz em doentes que apresentaram dor radicular. Entretanto, deve ser limitada no tratamento da dor perineal, provavelmente devido a limitações anatômicas, pois a inervação local tem caráter diferenciado, bem como as limitações técnicas para atingir essa cadeia nervosa.

Conclui-se que há resultados satisfatórios para o uso da SCS no tratamento da FBSS. Contudo, devem ser conduzidos mais estudos para determinar sua efetividade em médio e longo prazos.

\section{Referências}

1. Shealy CN, Mortimer JT, Reswick JB. Electrical inhibition of pain by stimulation of the dorsal columns: preliminary clinical report. Anesth Analg. 1967;46(4):489-91.

2. de la Porte C, Siegfried J. Lumbosacral spinal fibrosis (spinal arachnoiditis). Its diagnosis and treatment by spinal cord stimulation. Spine (Phila Pa 1976). 1983;8(6):593-603.

3. Kumar K, Wyant GM, Ekong CE. Epidural spinal cord stimulation for relief of chronic pain. Pain Clin. 1986;1(2):91-9.

4. Long DM, Erickson DE. Stimulation of the posterior columns of the spinal cord for relief of intractable pain. Surg Neurol. 1975;4(1):134-41.

5. Meglio M, Cioni B, Rossi GF. Spinal cord stimulation in management of chronic pain. A 9-year experience. J Neurosurg. 1989;70(4):519-24.
6. Ray CD, Burton CV, Lifson A. Neurostimulation as used in a large clinical practice. Appl Neurophysiol. 1982;45(12):160-6.

7. Siegfried J, Lazorthes Y. Long-term follow-up of dorsal cord stimulation for chronic pain syndrome after multiple lumbar operations. Appl Neurophysiol. 1982;45(1-2):201-4.

8. Young RF. Evaluation of dorsal column stimulation in the treatment of chronic pain. Neurosurgery. 1978;3(3):373-9.

9. Melzack R, Wall PD. Pain mechanisms: a new theory. Science. 1965;150(3699):971-9.

10. Cook AW, Oygar A, Baggenstos P, Pacheco S, Kleriga E. Vascular disease of extremities. Electric stimulation of spinal cord and posterior roots. NY State J Med. 1976;76(3):366-8.

11. Kemler MA, Barendse GA, van Kleef M, de Vet HC, Rijks $\mathrm{CP}$, Furnée $\mathrm{CA}$, et al. Spinal cord stimulation in patients with chronic reflex sympathetic dystrophy. $\mathrm{N}$ Engl J Med. 2000;343(9):618-24.

12. Tesfaye S, Watt J, Benbow SJ, Pang KA, Miles J, MacFarlane IA. Electrical spinal-cord stimulation for painful diabetic peripheral neuropathy. Lancet. 1996;348(9043):1698-701.

13. North RB, Kidd DH, Lee MS, Piantodosi S. A prospective, randomized study of spinal cord stimulation versus reoperation for failed back surgery syndrome: initial results. Stereotact Funct Neurosurg. 1994;62(1-4):267-72.

14. Merskey $\mathrm{H}$, Bogduk N. Classification of chronic pain: descriptions of chronic pain syndromes and definitions of pain terms. $2^{\text {nd }}$ ed. Seattle: International Association for the Study of Pain; 1994.

15. Martin BI, Mirza SK, Comstock BA, Gray DT, Kreuter W, Deyo RA. Are lumbar spine reoperation rates falling with greater use of fusion surgery and new surgical technology? Spine (Phila Pa 1976). 2007;32(19):2119-26.

16. Hunter C, Davé N, Diwan S, Deer T. Neuromodulation of pelvic visceral pain: review of the literature and case series of potential novel targets for treatment. Pain Pract. 2013;13(1):3-17.

17. De Andres J, Perotti L, Villaneuva-Perez VL, AsensioSamper JM, Fabregat-Cid G. Role of lumbosacral retrograde neuromodulation in the treatment of painful disorders. Pain Physician. 2013;16(2):145-53.

18. North RB, Kidd DH, Farrokhi F, Piantadosi SA. Spinal cord stimulation versus repeated lumbosacral spine surgery for chronic pain: a randomized, controlled trial. Neurosurgery. 2005;56(1):98-106.

19. Blond S, Buisset N, Dam Hieu P, Nguyen JP, Lazorthes Y, Cantagrel N, et al. Cost-benefit evaluation of spinal cord stimulation treatment for failed-back surgery syndrome patients. Neurochirurgie. 2004;50(4):443-53.

20. Kumar K, Taylor RS, Jacques L, Eldabe S, Meglio M, Molet J, et al. Spinal cord stimulation versus conventional medical management for neuropathic pain: a multicentre randomised controlled trial in patients with failed back surgery syndrome. Pain. 2007;132(1-2):179-88.

21. Kinfe TM, Schu S, Quack FJ, Wille C, Vesper J. Percutaneous implanted paddle lead for spinal cord stimulation: technical considerations and long-term follow-up. Neuromodulation. 2012;15(4):402-7.

22. Mailis-Gagnon A, Furlan AD, Sandoval JA, Taylor R. Spinal cord stimulation for chronic pain. Cochrane Database Syst Rev. 2004;(3):CD003783.

23. Cameron T. Safety and efficacy of spinal cord stimulation for the treatment of chronic pain: a 20-year literature review. J Neurosurg. 2004;100(3 Suppl Spine):254-67.

24. Turner JA, Loeser JD, Deyo RA, Sanders SB. Spinal cord stimulation for patients with failed back surgery syndrome or complex regional pain syndrome: a systematic review 
of effectiveness and complications. Pain. 2004;108(12):137-47.

25. Middleton PM, Simpson B, Maddern G. Spinal cord stimulation (neurostimulation): an accelerated systematic review. ASERNIP-S Report $n^{\circ}$ 43. Adelaide: ASERNIP-S; 2003.

26. Coleman SD, Mackey S. Spinal cord stimulation compared with medical management for failed back surgery syndrome. Curr Pain Headache Rep. 2009;13(1):1-2.

27. Vonhögen LH, Vancamp T, Vanneste S, Pollet W, Dirksen $\mathrm{R}$, Bakker $\mathrm{P}$, et al. Percutaneously implanted plates in failed back surgery syndrome (FBSS). Neuromodulation. 2011;14(4):319-24.

28. Rigoard P, Delmotte A, D'Houtaud S, Misbert L, Diallo B, Roy-Moreau A, et al. Back pain: a real target for spinal cord stimulation? Neurosurgery. 2012;70(3):574-84.

29. Logé D, Vanneste S, Vancamp T, Rijckaert D. Long-term outcomes of spinal cord timulation with percutaneously introduced paddle leads in the treatment of failed back surgery syndrome and lumboischialgia. Neuromodulation. 2013;16(6):537-45.

30. Kumar K. Spinal cord stimulation versus conventional medical management: a multicentre randomised controlled trial of patients with failed back surgery syndrome (PROCESS study). In: Conference Proceedings of 5th Congress of the European Federation of the International Association for the Study of Pain (IASP) Chapters (EFIC). Pain in Europe V. Istanbul, Turkey September 13-16, 2006. (Abstract $n^{\circ} 458$ ).
31. Kumar K, Malik S, Demeria D. Treatment of chronic pain with spinal cord stimulation versus alternative therapies: costeffectiveness analysis. Neurosurgery. 2002;51(1):106-15.

32. Taylor RS, Van Buyten JP, Buchser E. Spinal cord stimulation for chronic back and leg pain and failed back surgery syndrome: a systematic review and analysis of prognostic factors. Spine (Phila Pa 1976). 2005;30(1):152-60.

33. North R, Shipley J, Prager J, Barolat G, Barulich M, Bedder $\mathrm{M}$, et al. Practice parameters for the use of spinal cord stimulation in the treatment of chronic neuropathic pain. Pain Med. 2007;8(Suppl 4):200-75.

34. Kumar K, Buchser E, Linderoth B, Meglio M, Van Buyten JP. Avoiding complications from spinal cord stimulation: practical recommendations from an international panel of experts. Neuromodulation. 2007;10(1):24-33.

35. Kumar K, Wilson JR. Factors affecting spinal cord stimulation outcome in chronic benign pain with suggestions to improve success rate. Acta Neurochir Suppl. 2007;97(Pt 1):91-9.

36. Carter ML. Spinal cord stimulation in chronic pain: a review of the evidence. Anaesth Intensive Care. 2004;32(1):11-21.

\section{Endereço para correspondência}

Rodrigo Almeida Matos

Rua Florianópolis, quadra 12/13, 428, ap. 1104

Edifício Monte Castelo Setor Alto da Glória

75815-770 - Goiânia, GO, Brasil

Telefones: (62) 3204-6325/(62) 8450-7000

E-mail: mattos.ro@hotmail.com 